\title{
Well-posedness of a parabolic equation with nonlocal boundary condition
}

\author{
Allaberen Ashyralyev ${ }^{1,2}$ and Abdizhahan Sarsenbi $i^{3,4^{*}}$
}

${ }^{\text {*Correspondence: }}$
abzhahan@gmail.com
${ }^{3}$ Department of Mathematical
Methods and Modeling, M. Auezov
SKS University, Shimkent,
Kazakhstan
${ }^{4}$ Institute of Mathematics and
Mathematical Modeling, Almaty,
Kazakhstan
Full list of author information is
available at the end of the article

${ }^{\text {*Correspondence: }}$

abzhahan@gmail.com

Methods and Modeling, M. Auezov

University, Shimkent,

${ }^{4}$ Institute of Mathematics and

Mathematical Modeling, Almaty,

Full list of author information is

avalable at the end of the article

\begin{abstract}
In the present study, the problem of a parabolic equation with nonlocal condition is investigated. The positivity of the second order differential operator $A^{x}$ defined by the formula

$$
A^{x} u(x)=-u^{\prime \prime}(x)+\sigma u(x), \quad x \in(0,1)
$$
\end{abstract}

with the domain

$$
D\left(A^{x}\right)=\left\{u: u, u^{\prime \prime} \in C[0, I], u(0)=0, u^{\prime}(0)=u^{\prime}(I)+\beta u(I)\right\}
$$

is established in the Banach space $C[0, /]$ of all continuous functions $\phi(x)$ defined on $[0,1]$ with the norm

$$
\|\phi\|_{C[0, \eta]}=\max _{0 \leq x \leq 1}|\phi(x)|
$$

The well-posedness of this problem in Hölder spaces in $t$ is proved.

Keywords: parabolic equation; Banach spaces; positive operators; stability estimates; nonlocal condition

\section{Introduction}

Parabolic equations have important applications in a wide range of fields such as physics, chemistry, biology, ecology, and other. In mathematical modeling, parabolic equations are used together with boundary conditions specifying the solution on the boundary of the domain. Dirichlet and Neumann conditions are examples of classical boundary conditions (see, for example, [1] and the references therein). In some cases, classical boundary conditions cannot describe a process or a phenomenon precisely. Therefore, mathematical models of various physical, chemical, biological, or environmental processes often involve nonclassical conditions. Such conditions usually are identified as nonlocal boundary conditions and reflect situations when the data on the domain boundary cannot be measured directly, or when the data on the boundary depend on the data inside the domain. The well-posedness of various nonlocal boundary value problems for partial differential and difference equations has been studied extensively by many researchers (see, e.g., [1$20]$ and the references given therein). However, the well-posedness of the problem for 
a parabolic equation with nonlocal boundary conditions is not well investigated. In the present paper, we study the initial boundary value problem for a parabolic equation with nonlocal condition

$$
\left\{\begin{array}{l}
\frac{\partial u(t, x)}{\partial t}=a(x) \frac{\partial^{2} u(t, x)}{\partial x^{2}}-\sigma u(t, x)+f(t, x), \quad 0<x<l, 0<t<T, \\
u(t, 0)=0, \quad u_{x}(t, 0)=u_{x}(t, l)+\beta u(t, l), \quad 0 \leq t \leq T, \\
u(0, x)=\varphi(x), \quad 0 \leq x \leq l,
\end{array}\right.
$$

where $u(t, x)$ is an unknown function, $\varphi(x), a(x)$, and $f(t, x)$ are sufficiently smooth functions, $a(x) \geq \delta>0, \beta \geq 0$, and $\sigma>0$ is a sufficiently large number.

In the paper [21], initial boundary value problem (1) in the special case $a(x)=1$, $f(t, x)=0, \sigma=0$ was investigated by the separation of variables method. For this special case, we have the following spectral problem:

$$
\left\{\begin{array}{l}
-\frac{d^{2} v(x)}{d x^{2}}+\lambda v(x)=0, \quad 0<x<l \\
v(0)=0, \quad v^{\prime}(0)=v^{\prime}(l)+\beta v(l) .
\end{array}\right.
$$

It is well known that the differential operator $A_{0}$ defined by the formula

$$
A_{0} v=-\frac{d^{2} v}{d x^{2}}
$$

with the domain $D\left(A_{0}\right)=\left\{v \in C^{(2)}[0, l]: v(0)=0, v^{\prime}(0)=v^{\prime}(l)+\beta v(l)\right\}$ is not a self-adjoint and positive definite operator. Moreover, in the paper [22], it was proved that the system of eigenfunctions generated by this operator has no basis property in $L_{2}[0, l]$. In the paper [21], a special system of functions was proposed to expand this mixed problem by means of a slight modification of the separation of variables method. Finally, for the solution of this problem, a priori estimate in the norm of space $C\left([0, T], L_{2}[0, l]\right)$ was obtained. Note that the complete basis property of eigenfunctions of differential operators with constant coefficients and its applications have been studied by many scientists (see, e.g., [23-32] and the references given therein). Nevertheless, in a general case, the separation of variables method is not applicable.

In the present study, the well-posedness of a classical solution of problem (1) for a parabolic equation with nonlocal condition is investigated by the operator tool of monograph [33]. The stability estimates in $C([0, T], C[0, l])$ norm and coercive stability estimates in $C^{\alpha}([0, T], C[0, l])$ and $C_{0}^{\alpha}([0, T], C[0, l])$ norms for the solution of this problem are established.

\section{Preliminaries. Main results}

In the present paper, $C^{\alpha}([0, T], E)$ and $C_{0}^{\alpha}([0, T], E)(0<\alpha<1)$ stand for Banach spaces of all abstract continuous functions $\varphi(t)$ defined on $[0, T]$ with values in $E$ satisfying a Hölder condition for which the following norms are finite:

$$
\begin{aligned}
& \|\varphi\|_{C^{\alpha}([0, T], E)}=\|\varphi\|_{C([0, T], E)}+\sup _{0 \leq t<t+\tau \leq T} \frac{\|\varphi(t+\tau)-\varphi(t)\|_{E}}{\tau^{\alpha}}, \\
& \|\varphi\|_{C_{0}^{\alpha}([0, T], E)}=\|\varphi\|_{C([0, T], E)}+\sup _{0 \leq t<t+\tau \leq T} \frac{(t+\tau)^{\alpha}\|\varphi(t+\tau)-\varphi(t)\|_{E}}{\tau^{\alpha}},
\end{aligned}
$$


respectively. Here, $C([0, T], E)$ stands for the Banach space of all abstract continuous functions $\varphi(t)$ defined on $[0, T]$ with values in $E$ equipped with the norm

$$
\|\varphi\|_{C([0, T], E)}=\max _{0 \leq t \leq T}\|\varphi(t)\|_{E^{*}}
$$

Definition 2.1 An operator $A$ densely defined in a Banach space $E$ with domain $D(A)$ is called positive in $E$ if its spectrum $\sigma_{A}$ lies in the interior of the sector of angle $\varphi, 0<\varphi<\pi$, symmetric with respect to the real axis; moreover, on the edges of this sector $S_{1}(\varphi)=\left\{\rho e^{i \varphi}\right.$ : $0 \leq \rho \leq \infty\}$ and $S_{2}(\varphi)=\left\{\rho e^{-i \varphi}: 0 \leq \rho \leq \infty\right\}$ and outside of the sector the resolvent $(\lambda-$ $A)^{-1}$ is subject to the bound (see [33])

$$
\left\|(A-\lambda)^{-1}\right\|_{E \rightarrow E} \leq \frac{M}{1+|\lambda|}
$$

The infimum of all such angles $\varphi$ is called the spectral angle of the positive operator $A$ and is denoted by $\varphi(A)=\varphi(A, E)$. The operator $A$ is said to be strongly positive in a Banach space $E$ if $\varphi(A, E)<\frac{\pi}{2}$.

Throughout the present paper, we denote by $M$ positive constants which can be different from time to time and we are not interested in precise values. We will write $M(\alpha, \beta, \ldots)$ to stress the fact that the constant depends only on $\alpha, \beta, \ldots$.

With the help of the positive operator $A$, we introduce the fractional spaces $E_{\mu}=$ $E_{\mu}(E, A), 0<\mu<1$, consisting of all $v \in E$ for which the following norm is finite [33]:

$$
\|v\|_{E_{\mu}}=\|v\|_{E}+\sup _{\lambda>0} \lambda^{1-\mu}\|A \exp \{-\lambda A\} v\|_{E}
$$

To formulate our results, we introduce the Banach space $C[0, l]$ of all continuous functions $\phi(x)$ defined on $[0, l]$ with the norm

$$
\|\phi\|_{C[0, l]}=\max _{0 \leq x \leq l}|\phi(x)| .
$$

Finally, we introduce a differential operator $A^{x}$ defined by the formula

$$
A^{x} u=-a(x) \frac{d^{2} u}{d x^{2}}+\sigma u
$$

with the domain $D\left(A^{x}\right)=\left\{u \in C^{(2)}[0, l]: u(0)=0, u^{\prime}(0)=u^{\prime}(l)+\beta u(l)\right\}$.

We can rewrite problem (1) in the following abstract form:

$$
\left\{\begin{array}{l}
u_{t}(t)+A u(t)=f(t), \quad 0<t<T \\
u(0)=\varphi
\end{array}\right.
$$

in a Banach space $E=C[0, l]$ with the unbounded operator $A=A^{x}$ defined by formula (4). Here, $f(t)=f(t, x)$ and $u(t)=u(t, x)$ are, respectively, known and unknown abstract functions defined on $(0, T)$ with values in $E=C[0, l], \varphi=\varphi(x)$ and $a=a(x)$ are given elements of $E=C[0, l]$. 
The main result of the present paper is the following theorem on stability estimates of (1) in spaces $C([0, T], C[0, l])$ and coercive stability estimates in $C^{\alpha}([0, T], C[0, l])$ and $C_{0}^{\alpha}([0, T], C[0, l])$ norms for the solution of this problem.

Theorem 2.1 Assume that $\beta \geq 0, \varphi(x), \varphi_{x x}(x) \in C[0, l]$ and $f(t, x) \in C_{0}^{\alpha}([0, T], C[0, l])$. Then, for the solution of problem (1), the following stability estimate

$$
\|u\|_{C([0, T], C[0, l])} \leq M(\delta, \sigma, \alpha, l)\left[\|\varphi\|_{C[0, l]}+\|f\|_{C([0, T], C[0, l])}\right]
$$

and coercive stability estimate

$$
\begin{aligned}
& \left\|u_{t}\right\|_{C_{0}^{\alpha}([0, T], C[0, l])}+\left\|u_{x x}\right\|_{C_{0}^{\alpha}([0, T], C[0, l])} \\
& \quad \leq M(\delta, \sigma, \alpha, \beta, l)\left[\left\|\varphi_{x x}\right\|_{C[0, l]}+\|f\|_{C_{0}^{\alpha}([0, T], C[0, l])}\right]
\end{aligned}
$$

hold.

Theorem 2.2 Assume that $\beta \geq 0, \varphi(x), \varphi_{x x}(x) \in C[0, l]$ and

$$
-a(x) \varphi_{x x}(x)+\sigma \varphi(x)-f(0, x)=0
$$

and $f(t, x) \in C^{\alpha}([0, T], C[0, l])$. Then, for the solution of problem (1), the following coercive stability estimate

$$
\left\|u_{t}\right\|_{C^{\alpha}([0, T], C[0, l])}+\left\|u_{x x}\right\|_{C^{\alpha}([0, T], C[0, l])} \leq M(\delta, \sigma, \alpha, \beta, l)\|f\|_{C_{0}^{\alpha}([0, T], C[0, l])}
$$

holds.

The proofs of Theorems 2.1 and 2.2 are based on the following abstract theorem on the stability of problem (5) in $C([0, T], E)$ space and coercive stability in $C^{\alpha}([0, T], E)$ and $C_{0}^{\alpha}([0, T], E)$ spaces and on strong positivity of the unbounded operator $A=A^{x}$ defined by formula (4) in $C[0, l]$ space.

Theorem 2.3 [33] Let $A$ be a strongly positive operator in a Banach space $E$ and $f \in$ $C_{0}^{\alpha}([0, T], E)(0<\alpha<1)$. Then, for the solution of initial value problem (5), the stability and coercive stability inequalities

$$
\begin{aligned}
& \|u\|_{C([0, T], E)} \leq M\left[\|\varphi\|_{E}+\|f\|_{C([0, T], E)}\right], \\
& \left\|u^{\prime}\right\|_{C_{0}^{\alpha}([0, T], E)}+\|A u\|_{C_{0}^{\alpha}([0, T], E)} \\
& \quad \leq M\left[\|A \varphi\|_{E}+\frac{1}{\alpha(1-\alpha)}\|f\|_{C\left([0, T], E_{\beta}\right)}\right]
\end{aligned}
$$

hold. Moreover, assume that

$$
A \varphi-f(0)=0
$$


and $f \in C^{\alpha}([0, T], E)(0<\alpha<1)$. Then, for the solution of initial value problem (5), the coercive stability inequality

$$
\left\|u^{\prime}\right\|_{C^{\alpha}([0, T], E)}+\|A u\|_{C^{\alpha}([0, T], E)} \leq \frac{M}{\alpha(1-\alpha)}\|f\|_{C^{\alpha}([0, T], E)}
$$

holds.

In the next section, the Green's function of the operator $A=A^{x}$ defined by formula (4) is studied. The strong positivity of this operator in $C[0, l]$ space is established.

\section{The Green's function and strong positivity of $A^{x}$}

Lemma 3.1 Assume that $\varphi(x) \in C[0, l]$ and $\beta \geq 0$. Then, for any $\lambda \geq 0$ and $\sigma>0$, the spectral problem

$$
\left\{\begin{array}{l}
-\frac{d^{2} v(x)}{d x^{2}}+(\lambda+\sigma) v(x)=\psi(x), \quad 0<x<l, \\
v(0)=0, \quad v^{\prime}(0)=v^{\prime}(l)+\beta v(l)
\end{array}\right.
$$

is uniquely solvable, and the following formula holds:

$$
v(x)=\left(\lambda+A^{x}\right)^{-1} \psi(x)=\int_{0}^{l} G(x, s ; \lambda) \psi(s) d s,
$$

where

$$
\begin{aligned}
& G(x, s ; \lambda)=\left(1-e^{-2 l b}\right)^{-1} \\
& \times\left\{\begin{array}{cc}
\left(e^{-(l-x) b}-e^{-(l+x) b}\right) P\left(e^{-(l-s) b}+e^{-s b}\right) & \\
\quad+\left(e^{-(x-s) b}-e^{-(x+s) b}\right)\left(1-e^{-(2 l-2 x) b}\right) \times(2 b)^{-1}, & 0 \leq s \leq x, \\
\left(e^{-(l-x) b}-e^{-(l+x) b}\right) P\left(e^{-(l-s) b}+e^{-s b}\right) & \\
\quad+\left(e^{-(s-x) b}-e^{-(x+s) b}\right)\left(1-e^{-(2 l-2 s) b}\right) \times(2 b)^{-1}, & x<s \leq l,
\end{array}\right.
\end{aligned}
$$

where $P=\left(b+\beta+(-b+\beta) e^{-l b}\right)^{-1}$ and $b=\sqrt{\lambda+\sigma}$.

Proof It is clear that (see [34]) the boundary value problem for the second order differential equation

$$
\left\{\begin{array}{l}
-\frac{d^{2} v}{d x^{2}}+(\lambda+\sigma) v(x)=\psi(x), \quad 0<x<l, \\
v(0)=0, \quad v(l)=\omega
\end{array}\right.
$$

has a unique solution

$$
\begin{aligned}
v(x)= & \left(1-e^{-2 l b}\right)^{-1}\left\{\left(e^{-(l-x) b}-e^{-(l+x) b}\right) \omega\right. \\
& \left.-\left(e^{-(l-x) b}-e^{-(l+x) b}\right)(2 b)^{-1} \int_{0}^{l}\left(e^{-(l-s) b}-e^{-(l+s) b}\right) \psi(s) d s\right\} \\
& +(2 b)^{-1} \int_{0}^{l}\left(e^{-|x-s| b}-e^{-(x+s) b}\right) \psi(s) d s .
\end{aligned}
$$


We have that

$$
\begin{aligned}
v^{\prime}(x)= & \left(1-e^{-2 l b}\right)^{-1}\left\{b\left(e^{-(l-x) b}+e^{-(l+x) b}\right) \omega\right. \\
& \left.-\left(e^{-(l-x) b}+e^{-(l+x) b}\right) 2^{-1} \int_{0}^{l}\left(e^{-(l-s) b}-e^{-(l+s) b}\right) \psi(s) d s\right\} \\
& -2^{-1} \int_{0}^{x} e^{-(x-s) b} \psi(s) d s+2^{-1} \int_{x}^{l} e^{-(s-x) b} \psi(s) d s \\
& +2^{-1} \int_{0}^{l} e^{-(x+s) b} \psi(s) d s .
\end{aligned}
$$

Using formulas (12), (13) and the nonlocal boundary condition $v^{\prime}(0)=v^{\prime}(l)+\beta v(l)$, we get

$$
\begin{aligned}
(1- & \left.e^{-2 l b}\right)^{-1}\left\{2 b e^{-l b} \omega-e^{-l b} \int_{0}^{l}\left(e^{-(l-s) b}-e^{-(l+s) b}\right) \psi(s) d s\right\}+\int_{0}^{l} e^{-s b} \psi(s) d s \\
= & \left(1-e^{-2 l b}\right)^{-1}\left\{b\left(1+e^{-2 l b}\right) \omega-\left(1+e^{-2 l b}\right) 2^{-1} \int_{0}^{l}\left(e^{-(l-s) b}-e^{-(l+s) b}\right) \psi(s) d s\right\} \\
& -2^{-1} \int_{0}^{l} e^{-(l-s) b} \psi(s) d s+2^{-1} \int_{0}^{l} e^{-(l+s) b} \psi(s) d s+\beta \omega .
\end{aligned}
$$

Since $b+\alpha+(-b+\alpha) e^{-l b} \neq 0$, we have that

$$
\omega=P\left(1-e^{-l b}\right)^{-1} \int_{0}^{l}\left(-e^{-(2 l-s) b}-e^{-(l+s) b}+e^{-(l-s) b}+e^{-s b}\right) \psi(s) d s .
$$

Consequently, if the function $\psi$ is continuously differentiable on $[0, l]$, then formulas (12), (14) give a solution of problem (8). Applying formulas (12), (14), we get

$$
\begin{aligned}
v(x)= & \left(1-e^{-2 l b}\right)^{-1}\left\{\left(e^{-(l-x) b}-e^{-(l+x) b}\right)\right. \\
& \times P\left(1-e^{-l b}\right)^{-1} \int_{0}^{l}\left(-e^{-(2 l-s) b}-e^{-(l+s) b}+e^{-(l-s) b}+e^{-s b}\right) \psi(s) d s \\
& \left.-\left(e^{-(l-x) b}-e^{-(l+x) b}\right)(2 b)^{-1} \int_{0}^{l}\left(e^{-(l-s) b}-e^{-(l+s) b}\right) \psi(s) d s\right\} \\
& +(2 b)^{-1} \int_{0}^{l}\left(e^{-|x-s| b}-e^{-(x+s) b}\right) \psi(s) d s \\
= & \int_{0}^{l} G(x, s ; \lambda) \psi(s) d s,
\end{aligned}
$$

where

$$
\begin{aligned}
G(x, s ; \lambda)= & \left(1-e^{-2 l b}\right)^{-1}\left\{\left(e^{-(l-x) b}-e^{-(l+x) b}\right) P\left(e^{-(l-s) b}+e^{-s b}\right)\right. \\
& -\left(e^{-(l-x) b}-e^{-(l+x) b}\right)(2 b)^{-1}\left(e^{-(l-s) b}-e^{-(l+s) b}\right) \\
& \left.+(2 b)^{-1}\left(1-e^{-2 l b}\right)\left(e^{-|x-s| b}-e^{-(x+s) b}\right)\right\} .
\end{aligned}
$$

From that, formula (10) follows. Lemma 3.1 is proved. 
Note that formula (10) is true for some $\lambda \in \mathbb{C}$. Assume that $\lambda=|\lambda| e^{ \pm \varphi}, \varphi \in\left[0, \frac{\pi}{2}\right]$ and $\beta \geq 0, \sigma>0$. Then we have that the following estimate holds:

$$
\left|\frac{-b+\beta}{b+\beta}\right| \leq 1, \quad|P| \leq \frac{1}{\sqrt[4]{\sigma^{2}+|\lambda|^{2}}\left(1-e^{-\sqrt[4]{\sigma^{2}+|\lambda|^{2}} \frac{\sqrt{2}}{2}}\right)} .
$$

Therefore, the following pointwise estimates for $G(x, s ; \lambda)$ hold:

$$
|G(x, s ; \lambda)| \leq \frac{M(\delta, \sigma, l, \beta)}{\sqrt[4]{\sigma^{2}+|\lambda|^{2}}} \begin{cases}e^{-(l-x+s) \sqrt[4]{\sigma^{2}+|\lambda|^{2}} \frac{\sqrt{2}}{2}}, & 0 \leq s \leq x-\frac{l}{2} \\ e^{-(x-s) \sqrt[4]{\sigma^{2}+|\lambda|^{2}} \frac{\sqrt{2}}{2}}, & x-\frac{l}{2} \leq s \leq x \\ e^{-(s-x) \sqrt[4]{\sigma^{2}+|\lambda|^{2}} \frac{\sqrt{2}}{2}}, & x \leq s \leq x+\frac{l}{2} \\ e^{-(l+x-s) \sqrt[4]{\sigma^{2}+|\lambda|^{2}} \frac{\sqrt{2}}{2}}, & x+\frac{l}{2} \leq s \leq l\end{cases}
$$

Theorem 3.1 Assume that $a(x)$ satisfies a Hölder condition on the segment $[0, l]$ and $a(x) \geq$ $\delta>0$. Then the operator $A=A^{x}$ defined by formula (4) is a strongly positive operator in $C[0, l]$ space.

Proof First, we prove this statement for the differential operator $A^{x}$ defined by formula (4) in the case when $a(x)=1$. Applying the triangle inequality and formula (9), we get

$$
\left|\left(\lambda+A^{x}\right)^{-1} \psi(x)\right| \leq \int_{0}^{l}|G(x, s ; \lambda)||\psi(s)| d s \leq \int_{0}^{l}|G(x, s ; \lambda)| d s\|\psi\|_{C[0, l]}
$$

for any $x \in[0, l]$. Therefore,

$$
\left\|\left(\lambda+A^{x}\right)^{-1} \psi\right\|_{C[0, l]} \leq \max _{0 \leq x \leq l} \int_{0}^{l}|G(x, s ; \lambda)| d s\|\psi\|_{C[0, l]},
$$

which means that

$$
\left\|\left(\lambda+A^{x}\right)^{-1}\right\|_{C[0, l] \rightarrow C[0, l]} \leq \max _{0 \leq x \leq l} \int_{0}^{l}|G(x, s ; \lambda)| d s .
$$

Applying the triangle inequality and estimate (15), we get

$$
\max _{0 \leq x \leq l} \int_{0}^{l}|G(x, s ; \lambda)| d s \leq \frac{M(\delta, \sigma, l, \beta)}{\sqrt{\sigma^{2}+|\lambda|^{2}}} \leq \frac{M_{1}(\delta, \sigma, l, \beta)}{1+|\lambda|} .
$$

Therefore,

$$
\left\|\left(\lambda+A^{x}\right)^{-1}\right\|_{C[0, l] \rightarrow C[0, l]} \leq \frac{M_{1}(\delta, \sigma, l, \beta)}{1+|\lambda|} .
$$

It is easy to see that if in the operator $A^{x}$ the coefficient $a(x)=a=$ const, then the resolvent equation

$$
\left\{\begin{array}{l}
-a(x) \frac{d^{2} v(x)}{d x^{2}}+(\lambda+\sigma) v(x)=\psi(x), \quad 0<x<l, \\
v(0)=0, \quad v^{\prime}(0)=v^{\prime}(l)+\beta v(l)
\end{array}\right.
$$


can be transformed in the form (8) by dividing both sides of the resolvent equation (18) to $a$. We have the following estimates for the Green's function:

$$
\left|G^{x}(x, s ; \lambda)\right| \leq \frac{M(\delta, \sigma, l, \beta)}{\sqrt[4]{\sigma^{2}+|\lambda|^{2}}} \begin{cases}e^{-\frac{(l-x+s)}{\sqrt{\delta}} \sqrt[4]{\sigma^{2}+|\lambda|^{2}} \frac{\sqrt{2}}{2}}, & 0 \leq s \leq x-\frac{l}{2} \\ e^{-\frac{(x-s)}{\sqrt{\delta}} \sqrt[4]{\sigma^{2}+|\lambda|^{2}} \frac{\sqrt{2}}{2}}, & x-\frac{l}{2} \leq s \leq x \\ e^{-\frac{(s-x)}{\sqrt{\delta}} \sqrt[4]{\sigma^{2}+|\lambda|^{2}} \frac{\sqrt{2}}{2}}, & x \leq s \leq x+\frac{l}{2} \\ e^{-\frac{(l+x-s)}{\sqrt{\delta}} \sqrt[4]{\sigma^{2}+|\lambda|^{2}} \frac{\sqrt{2}}{2}}, & x+\frac{l}{2} \leq s \leq l\end{cases}
$$

Therefore, applying the triangle inequality, estimate (19), we get estimate (17).

Second, let $a(x)$ satisfy a Hölder condition on the segment $[0, l]$ and $a(x) \geq \delta>0$.

We will use the method of frozen coefficients. Let $\varepsilon>0$ be an arbitrary small given number. From the compactness of $[0, l]$ it follows that there is a system of $\left\{I_{j}\right\}, j=1, \ldots, r$, of intervals and two half-intervals (containing 0 and $l$, resp.) covering the segment $[0, l]$ and such that $\left|a\left(x_{1}\right)-a\left(x_{2}\right)\right|<\varepsilon, x_{1}, x_{2} \in I_{j}$. Consider a partition of unity corresponding to this system, i.e., a system of smooth nonnegative functions $\xi_{j}(x)(i=1, \ldots, r)$ with supp $\xi_{j}(x) \subset I_{j}, \xi_{j}(0)=\xi_{j}(l), \xi_{j}^{\prime}(0)=0, \xi_{j}^{\prime}(l)=0$, and $\xi_{1}(x)+\cdots+\xi_{r}(x)=1$ in $[0, l]$.

Clearly, for the positivity of $A^{x}$ in $C[0, l]$, it is enough to establish the estimate (17) for the solution of problem (18). Let $w(x)=\xi_{j}(x) u(x)$. Then we have the following nonlocal boundary value problem:

$$
\left\{\begin{array}{l}
(\delta+\lambda) w(x)-a^{j} w^{\prime \prime}(x)=F^{j}(x), \quad 0<x<l \\
w(0)=0, \quad w^{\prime}(0)=w^{\prime}(l)+\beta w(l),
\end{array}\right.
$$

where $a^{j}=a\left(x^{j}\right)$ and

$$
F^{j}(x)=\xi_{j}(x) f(x)-a(x)\left(2 \xi_{j}^{\prime}(x) u^{\prime}(x)+\xi_{j}^{\prime \prime}(x) u(x)\right)-\left(a^{j}-a(x)\right) w^{\prime \prime}(x) .
$$

Since equation (20) is an equation with constant coefficients, we have the following estimates:

$$
\begin{aligned}
& (1+|\lambda|)\|w\|_{C[0, l]} \leq K(\varphi, \delta)\left\|F^{j}\right\|_{C[0, l]}, \quad \lambda \in R_{\varphi}, \\
& \left\|w^{\prime \prime}\right\|_{C[0, l]} \leq M(\varphi, \delta)\left\|F^{j}\right\|_{C[0, l]} .
\end{aligned}
$$

Using the definition of $I_{j}$ and the continuity of $a(x)$, as well as the smoothness of $\xi_{j}(x)$, we obtain

$$
\left\|F^{j}\right\|_{C[0, l]} \leq M(\varphi, \delta)\left(\|f\|_{C[0, l]}+\|u\|_{C[0, l]}+\left\|u^{\prime}\right\|_{C[0, l]}\right)+\varepsilon\left\|w^{\prime \prime}\right\|_{C[0, l]} \text {. }
$$

Assume that $0<\varepsilon<1 / M(\varphi, \delta)$, then from the last estimate it follows that

$$
\begin{gathered}
\left\|w^{\prime \prime}\right\|_{C[0, l]} \leq \frac{M(\varphi, \delta)}{1-\varepsilon M(\varphi, \delta)}\left[\|f\|_{C[0, l]}+\left\|u^{\prime}\right\|_{C[0, l]}+\|u\|_{C[0, l]}\right], \\
\left\|F^{j}\right\|_{C[0, l]} \leq \frac{M(\varphi, \delta)}{1-\varepsilon M(\varphi, \delta)}\left[\|f\|_{C[0, l]}+\left\|u^{\prime}\right\|_{C[0, l]}+\|u\|_{C[0, l]}\right] .
\end{gathered}
$$


From this and estimate (21) it follows that

$$
\begin{aligned}
& (1+|\lambda|)\|w\|_{C[0, l]} \\
& \quad \leq K(\varphi, \delta) \frac{M(\varphi, \delta)}{1-\varepsilon M(\varphi, \delta)}\left[\|f\|_{C[0, l]}+\left\|u^{\prime}\right\|_{C[0, l]}+\|u\|_{C[0, l]}\right] .
\end{aligned}
$$

Using the triangle inequality, we obtain

$$
\begin{aligned}
& \left\|u^{\prime \prime}\right\|_{C[0, l]} \leq K_{1}(\varphi, \delta)\left[\|f\|_{C[0, l]}+\left\|u^{\prime}\right\|_{C[0, l]}+\|u\|_{C[0, l]}\right] \\
& (1+|\lambda|)\|u\|_{C[0, l]} \leq M_{1}(\varphi, \delta)\left[\|f\|_{C[0, l]}+\left\|u^{\prime}\right\|_{C[0, l]}+\|u\|_{C[0, l]}\right] .
\end{aligned}
$$

Now, using Nirenberg's inequality, we can write

$$
\begin{aligned}
F & =\|f\|_{C[0, l]}+\left\|u^{\prime}\right\|_{C[0, l]}+\|u\|_{C[0, l]} \\
& \leq M_{2}(\varphi, \delta)\left[\|f\|_{C[0, l]}+\alpha^{-1}\left\|u^{\prime}\right\|_{C[0, l]}+\alpha\|u\|_{C[0, l]}\right] .
\end{aligned}
$$

Hence, for small $\alpha$, from the last inequality and estimate (22) it follows that

$$
F \leq M_{3}(\varphi, \delta)\left[\alpha^{-1}\|u\|_{C[0, l]}+\|f\|_{C[0, l]}\right]
$$

Therefore, from estimate (23) it follows that

$$
(1+|\lambda|)\|u\|_{C[0, l]} \leq M_{3}(\varphi, \delta)\left[\alpha^{-1}\|u\|_{C[0, l]}+\|f\|_{C[0, l]}\right]
$$

Hence, for all $\lambda$, we have

$$
|\lambda|>\frac{M_{3}(\varphi, \delta)}{\alpha}=K_{0}
$$

estimate (23) is proved. This concludes the proof of Theorem 3.1.

Note that the arguments of Theorem 3.1 are not applicable for the multidimensional operator $A^{x}$ because the coercive inequality (3) is absent. Note that estimate (17) for the differential operator $A^{x}$ defined by formula (4) can be proved without using coercive inequality (3) under supplementary assumption for $\sigma$. Actually, under supplementary assumption that $\sigma>0$ is a sufficiently large number, applying a fixed point theorem and estimate (19) and the formula

$$
G^{x}\left(x, x_{0} ; \lambda\right)=G^{x_{0}}\left(x, x_{0} ; \lambda\right)+(\lambda+\delta) \int_{0}^{l} G^{x_{0}}(x, y ; \lambda)\left(\frac{1}{a(y)}-\frac{1}{a\left(x_{0}\right)}\right) G^{y}\left(y, x_{0} ; \lambda\right) d y,
$$

we can obtain the following estimates:

$$
\left|G^{x}\left(x, x_{0} ; \lambda\right)\right| \leq \frac{M(\delta, \sigma, l, \beta)}{\sqrt[4]{\sigma^{2}+|\lambda|^{2}}} \begin{cases}e^{-\frac{\left(l-x+x_{0}\right)}{3 \sqrt{\delta}} \sqrt[4]{\sigma^{2}+|\lambda|^{2}} \frac{\sqrt{2}}{2}}, & 0 \leq x_{0} \leq x-\frac{l}{2} \\ e^{-\frac{\left(x-x_{0}\right)}{3 \sqrt{\delta}} \sqrt[4]{\sigma^{2}+|\lambda|^{2}} \frac{\sqrt{2}}{2}}, & x-\frac{l}{2} \leq x_{0} \leq x \\ e^{-\frac{\left(x_{0}-x\right)}{3 \sqrt{\delta}} \sqrt[4]{\sigma^{2}+|\lambda|^{2}} \frac{\sqrt{2}}{2}}, & x \leq x_{0} \leq x+\frac{l}{2} \\ e^{-\frac{\left(l+x-x_{0}\right)}{3 \sqrt{\delta}} \sqrt[4]{\sigma^{2}+|\lambda|^{2}} \frac{\sqrt{2}}{2}}, & x+\frac{l}{2} \leq x_{0} \leq l\end{cases}
$$


for the Green's function of the differential operator $A^{x}$ defined by formula (4). Therefore, the statement of Theorem 3.1 is true for the differential operator $A^{x}$ defined by formula (4).

\section{Conclusion}

In the present study, problem (1) for a parabolic equation with nonlocal condition is investigated. The stability and coercive estimates in Hölder norms for the solution of this problem are established.

Moreover, applying the result of the monograph [34], the high order of accuracy singlestep difference schemes for the numerical solution of problem (1) for a parabolic equation with nonlocal condition can be presented. Of course, the stability estimates for the solution of these difference schemes have been established without any assumptions about the grid steps. Moreover, basis properties for a system of root vectors of the corresponding generalized spectral problem with various boundary conditions for a wide class of operators have been investigated in papers [23-32]. Applying the method of the present paper, the stability estimates for the solution of partial differential equations with this class of operators can be established.

Competing interests

The authors declare that they have no competing interests.

Authors' contributions

All authors contributed equally to the writing of this paper. All authors read and approved the final manuscript.

\section{Author details}

'Department of Mathematics, Fatih University, Istanbul, Turkey. ${ }^{2}$ Department of Applied Mathematics, ITTU, Ashgabat, Turkmenistan. ${ }^{3}$ Department of Mathematical Methods and Modeling, M. Auezov SKS University, Shimkent, Kazakhstan. ${ }^{4}$ Institute of Mathematics and Mathematical Modeling, Almaty, Kazakhstan.

\section{Acknowledgements}

The authors are thankful to the honorable reviewers for their valuable suggestions and comments, which improved the paper. This work is supported by the Grant No. 5414/GF4 of the Committee of Science of Ministry of Education and Science of the Republic of Kazakhstan.

Received: 10 November 2014 Accepted: 28 January 2015 Published online: 24 February 2015

\section{References}

1. Sapagovas, M, Jakubelienè, K: Alternating direction method for two-dimensional parabolic equation with nonlocal integral condition. Nonlinear Anal., Model. Control 17(1), 91-98 (2012)

2. Wang, Y, Zheng, S: The existence and behavior of solutions for nonlocal boundary problems. Bound. Value Probl. 2009, Article ID 484879 (2009). doi:10.1155/2009/484879

3. Ashyralyev, A, Karatay, I, Sobolevskii, PE: On well-posedness of the nonlocal boundary value problem for parabolic difference equations. Discrete Dyn. Nat. Soc. 2, 273-286 (2004). doi:10.1155/S1026022604403033

4. Zouyed, F, Rebbani, F, Boussetila, N: On a class of multitime evolution equations with nonlocal initial conditions. Abstr. Appl. Anal. 2007, Article ID 16938 (2007). doi:10.1155/2007/16938

5. Boucherif, A, Precup, R: Semilinear evolution equations with nonlocal initial conditions. Dyn. Syst. Appl. 16(3), 507-516 (2007)

6. Jachimavičienè, J, Sapagovas, M, Štikonas, A, Štikonienè, O: On the stability of explicit finite difference schemes for a pseudoparabolic equation with nonlocal conditions. Nonlinear Anal., Model. Control 19(2), 225-240 (2014)

7. Čiupaila, R, Sapagovas, M, Štikonienè, O: Numerical solution of nonlinear elliptic equation with nonlocal condition. Nonlinear Anal., Model. Control 18(4), 412-426 (2013)

8. Ashyralyev, A: Nonlocal boundary-value problems for abstract parabolic equations: well-posedness in Bochner spaces. J. Evol. Equ. 6(1), 1-28 (2006). doi:10.1007/s00028-005-0194-y

9. Ivanauskas, F, Meškauskas, T, Sapagovas, M: Stability of difference schemes for two-dimensional parabolic equations with non-local boundary conditions. Appl. Math. Comput. 215(7), 2716-2732 (2009)

10. Ivanauskas, FF, Novitski, YA, Sapagovas, M: On the stability of an explicit difference scheme for hyperbolic equations with nonlocal boundary conditions. Differ. Equ. 49(7), 849-856 (2013)

11. Sajavičius, S: Stability of the weighted splitting finite-difference scheme for a two-dimensional parabolic equation with two nonlocal integral conditions. Comput. Math. Appl. 64(11), 3485-3499 (2012)

12. Ashyralyyev, C, Dural, A, Sozen, Y: Finite difference method for the reverse parabolic problem. Abstr. Appl. Anal. 2012, Article ID 294154 (2012). doi:10.1155/2012/294154

13. Ashyralyev, A, Sobolevskii, PE: Difference schemes of the high order accuracy for parabolic equations with variable-coefficients. Dokl. Akad. Nauk Ukr. SSR, Ser. A. Fiz.-Mat. Teh. Nauki 6, 3-7 (1988) 
14. Ashyralyev, A, Ozdemir, Y: On nonlocal boundary value problems for hyperbolic-parabolic equations. Taiwan. J. Math. 11(4), 1075-1089 (2007)

15. Ashyraliyev, M: A note on the stability of the integral-differential equation of the parabolic type in a Banach space. Abstr. Appl. Anal. 2012, Article ID 178084 (2012). doi:10.1155/2012/178084

16. Sahmurova, A, Shakhmurov, VB: Parabolic problems with parameter occurring in environmental engineering. In: First International Conference on Analysis and Applied Mathematics: ICAAM 2012. AIP Conference Proceedings, vol. 1470, pp. 39-41 (2012)

17. Ashyralyev, A, Agirseven, D: Note on the stability of delay parabolic equations with unbounded operators acting on delay terms. Electron. J. Differ. Equ. 2014, 160 (2014)

18. Ashyralyev, A, Agirseven, D: Well-posedness of delay parabolic equations with unbounded operators acting on delay terms. Bound. Value Probl. 2014, Article ID 126 (2014). doi:10.1186/1687-2770-2014-126

19. Ashyralyev, A, Agirseven, D: On source identification problem for a delay parabolic equation. Nonlinear Anal., Model. Control 19(3), 335-349 (2014)

20. Ashyralyev, A: A note on the Bitsadze-Samarskii type nonlocal boundary value problem in a Banach space. J. Math. Anal. Appl. 344(1), 557-573 (2008). doi:10.1016/j.jmaa.2008.03.008

21. Mokin, AY: Correctness of task family with nonclassical boundary conditions. Comput. Res. Model. 1(2), 139-146 (2009) (in Russian)

22. Il'in, VA: Basis property and equiconvergence with the trigonometric series of root function expansions and the form of boundary conditions for a nonself-adjoint differential operator. Differ. Equ. 30(9), 1402-1413 (1994)

23. Sadybekov, MA, Sarsenbi, AM: Criterion for the basis property of the eigenfunction system of a multiple differentiation operator with an involution. Differ. Equ. 48(8), 1112-1118 (2012)

24. Sarsenbi, AM, Tengaeva, AA: On the basis properties of root functions of two generalized eigenvalue problems. Differ. Equ. 48(2), 306-308 (2012)

25. Sadybekov, MA, Sarsenbi, AM: Mixed problem for a differential equation with involution under boundary conditions of general form. In: Ashyralyev, A, Lukashov, A (eds.) First International Conference on Analysis and Applied Mathematics: ICAAM 2012. AIP Conference Proceedings, vol. 1470, pp. 225-227 (2012)

26. Kopzhassarova, A, Sarsenbi, A: Basis properties of eigenfunctions of second-order differential operators with involution. Abstr. Appl. Anal. 2012, Article ID 576843 (2012)

27. Kopzhassarova, AA, Lukashov, AL, Sarsenbi, AM: Spectral properties of non-self-adjoint perturbations for a spectral problem with involution. Abstr. Appl. Anal. 2012, Article ID 590781 (2012)

28. Kal'menov, TS, Sadybekov, MA, Sarsenbi, AM: Biorthogonal expansions in root functions of differential operators. Differ. Equ. 47(1), 144-148 (2011)

29. Sarsenbi, AM: Unconditional bases related to a nonclassical second-order differential operator. Differ. Equ. 46(4), 509-514 (2010)

30. Sadybekov, MA, Sarsenbi, AM: On the theory of anti-a priori estimates in the sense of V.A. Il'in. Dokl. Math. 77(3), 398-400 (2008)

31. Sadybekov, MA, Sarsenbi, AM: The use of anti - a priori estimates in the theory of bases in the space $L_{2}$. Differ. Equ. 44(5), 685-691 (2008)

32. Sarsenbi, AM: Necessary and sufficient conditions for the Riesz basis property of the eigen- and associated functions of high-order differential operators on an interval. Dokl. Math. 77(2), 290-292 (2008)

33. Ashyralyev, A, Sobolevskii, PE: Well-Posedness of Parabolic Difference Equations. Operator Theory: Advances and Applications. Birkhäuser, Basel (1994)

34. Ashyralyev, A, Sobolevskii, PE: New Difference Schemes for Partial Differential Equations. Operator Theory: Advances and Applications. Birkhäuser, Basel (2004)

\section{Submit your manuscript to a SpringerOpen ${ }^{\odot}$ journal and benefit from:}

- Convenient online submission

Rigorous peer review

- Immediate publication on acceptance

- Open access: articles freely available online

- High visibility within the field

- Retaining the copyright to your article 